\title{
Recent Advances in Delivery of Antifungal Agents - A Review
}

\author{
Sasikala Chinnappan*, Chia Le Yi, Chow Jie Chen, Tan Wei Hsia, Yap Hui Qi \\ Faculty of Pharmaceutical Sciences, UCSI University, Kuala Lumpur, MALAYSIA.
}

\begin{abstract}
Drug delivery systems demonstrate how the pharmaceutical product is administered to the target site to provide therapeutic action. Nowadays, there are a growing number of fungi causing diseases worldwide. These infections can be grouped into four, which are superficial mycosis, cutaneous mycosis, subcutaneous mycosis and systemic mycosis. Researchers believe that solely dependent on presently available antifungal compounds would not be sufficient to treat these mycoses. Introduction of a whole new antifungal drug acting to the target sites must undergo a long process of discovery, various clinical testing and trials on animals and human, development and regulatory approval then finally brought to the market. It is tedious, big-budget and has a high probability of failure. Hence, concurrently, modifications of the existing drug delivery system have never left out and are being pursued innovatively. Applying the knowledge in pharmacodynamics and pharmacokinetic principles, novel advances in antifungal drug delivery systems have been developed and managed to conquer the issues of solubility, stability, bioavailability, safety and effectiveness present in conventional formulations and methods
\end{abstract}

of administration. Vesicular system, nano-particulate based system, colloidal carriers and the miscellaneous drug delivery system are the four major groups of advances in delivering antifungal drugs. The established therapeutic agents for antifungal therapy such as Amphotericin B and azole types can be formulated into different carriers and delivery systems that specifically target the infected area and the illness level of the patient. Novel antifungal drug delivery systems have opened a new dimension in minimising the adverse effects of drugs.

Key words: Fungal infections, Antifungal drugs, Novel delivery.

Correspondence

Dr. Sasikala Chinnappan

Assistance Professor, Faculty of Pharmaceutical sciences, UCSI University, Kuala Lumpur, MALAYSIA.

Phone: +60 186653642

Email: hasipharm@gmail.com

DOI: $10.5530 /$ jyp.2020.12.59

\section{INTRODUCTION}

Basically, there are about 300 out of 250,000 fungi species in world are implicated as potential or true pathogens that would cause superficial even life threatening infections in humans. ${ }^{1}$ However, most of the fungal infection are due to opportunistic pathogens such as Aspergillus, Candida and Cryptococcus spp. In other words, immunocompromised patients are more vulnerable to fungal infections as compared to healthy individuals, in which their immune system may be suppressed by drugs or weakened due to their medical conditions. For example, HIV/ AIDS, tuberculosis, cancer, diabetes mellitus, organ transplantation, trauma and even prolonged use of corticosteroid or antibiotics. ${ }^{1}$

Fungal infections lead to significant rates of mortality and morbidity. Drugs effective against fungal infections are available, but the challenges encountered are the evolution of drug resistance and high incidence of side effects, particularly toxicity caused by the antifungal agents. As there is only limited antifungal agents available, therefore the possible side effects caused by the antifungal agents will limit the dose and dosing frequency, therefore, resulting in lower efficacy and even therapeutic failure. ${ }^{2}$ For example, Amphotericin B which is choice of drug for the systemic mycoses, however it can induce moderate to severe nephrotoxicity which limits the use of this drug. Some antifungal agents which are delivered topically via formulations such as gel or cream may also cause irritation, swelling and redness on the site of application.

To address these problems, various approaches are developed to improve the therapeutic outcome. This paper will review the recent development in the delivery of antifungal agents to improve the treatment outcome and quality of patient's life. Novel drug delivery systems actually are designed to ensure the drug is delivered to the specific desired site at an extent and rate as directed by the needs of the patient and it can direct an active pharmaceutical ingredient to the specific site of action during the course of therapy. ${ }^{3}$ Recent advances in the drug delivery systems are significantly important by its improving patient acceptability and therapeutic efficacy which is achieved by alleviation of possible side effects and decreasing in dose required. In 1990s, the appearance of liposomal preparation of amphotericin B which marked the start of the use of nanostructured lipid system for antifungal therapy. ${ }^{4} \mathrm{Up}$ to date, there are different advances in antifungal drug delivery system which including vesicular system, nano-particulate based system, colloidal carriers and the miscellaneous drug delivery systems such as buccal mucoadhesive tablets. All these antifungal drug delivery systems have its own advantages and disadvantages and this will be further discussed in this review.

\section{VESICULAR DRUG DELIVERY SYSTEMS}

Vesicles can be defined as colloidal particles in which a concentric bilayer is formed by the amphiphilic building blocks surrounding an aqueous compartment. Vesicle can be used to deliver both hydrophilic and lipophilic drugs by encapsulation in the aqueous compartment or incorporated in the hydrophobic bilayer. ${ }^{5}$ Therefore, encapsulated drug is released via fusion of bilayer with the target cell membrane. Apart from that, the Vesicular Drug Delivery Systems (VDDS) are able to reduce the risk of drug toxicity as it plays a vital role in delivery of drugs to the targeted site such as specific organs or tissue for action thereby lowering its concentration on other sites of the body. Various advances have been made in VDDS such as designed to be degraded slowly to achieve sustained release of drugs and also to delay the elimination of rapid metabolized drugs. In addition, entrapment of drug in the 
vesicles is believed to lengthen the lifetime of drugs present in systemic circulation. ${ }^{6}$

\section{NANO-PARTICULATE BASED DRUG DELIVERY SYSTEM}

Nanoparticles are the particles with size within 10 to 1000 nanometre which used recently in the industry of pharmaceutical science due to there are many challenges in drug delivering of large sized materials. As in invasive mycoses treatment, nano structure is used to carry the antifungal drug to reduce its side effects and target their action. The nanostructure used in the delivery of antifungal drugs are liposome, solid nanoparticles and polymeric nanoparticles. In antifungal treatment, lipid formulations such as Amphotericin B or nystatin are used to reduce the toxicity.

Liposomes are a small artificial vesicle with spherical shape which consist of unilamellar or multilamellar layers. ${ }^{7}$ Because of liposomes have both lipophilic and hydrophilic characters, these increase the solubility of lipophilic drugs in aqueous body fluids and the hydrophilic drug penetration through phospholipid membranes. Liposomes are biodegradable, biocompatible and have a higher protection against enzymes compared with other lipid formulations. ${ }^{8}$

Solid lipid nanoparticles (SLNs) are a colloidal drug carrier which consist of surfactant-stabilized lipids that are solid at both room and body temperature. SLNs usually used in oral, parenteral and transdermal administration due to their good protection of active ingredients from degradation, good tolerability, controlled release and targeting of the specific site. ${ }^{9}$ The controlled release property makes SLNs a popular treatment of topical skin infections because of its prolonged release effect and high skin permeation. The lipophilic antifungal drug is entrapped within the solid lipidic core of SLNs. The drug released is fast and desirable by following the Fick's law of diffusion.

Polymeric nanoparticles are solid colloidal particles with size range $10 \mathrm{~nm}$ to 1 micrometer and can be classified into two according to their preparation method, which is nanosphere and nano capsule. A nanosphere is a matrix type structure in which the drug is uniformly distributed whereas a nano capsule has a membrane wall structure with an oil core containing the drug. Polymers used can be natural or synthetic in order to carry the drug to the target site.

\section{COLLOIDAL CARRIERS}

Microspheres are the active pharmaceutical ingredients homogeneously dispersed in the matrix system, whereas microcapsules are the drug reservoir and heterogeneousin nature. ${ }^{10}$ Microparticulates usually encoded by polymer excipients to protect drugs from degradation before reaching target tissues. The mechanism of drug released that made by biodegradable polymers varies from diffusion, dissolution, osmotically driven to erosion depends on the formulation and drug properties. Drug released is due to the contact of polymer coating drugs with biological fluid either by forming pores or the degradation of polymers. When drugs reached its specific target sites, fluids diffuse into or dissolve the polymers result in drug released. Erosion can be classified to surface or bulk erosion where the $\mathrm{pH}$ or enzyme causes hydrolysis reaction of the polymer coating leading to drug released. To facilitate the efficacy and therapeutic effect of antifungal drugs, certain methods are used to formulate and design the drugs. Econazole nitrate used in the treatment of vaginal candidiasis prepared in mucoadhesive microsphere formulation by spray-congealing method to sustain release and increase the duration of action. ${ }^{11}$ Poloxamers are the polymer that coated the econazole drug which increase the solubility and adhesion of econazole with mucous membrane. The drugs diffuse out slowly in the acidic $\mathrm{pH}$ of vagina. ${ }^{12}$
Microemulsions are thermodynamically stable and can be categorized as water in oil, oil in water or bicontinuous emulsion depends on the composition of oil phase, water phase, solvent and cosolvent. ${ }^{13}$ For instances, ketoconazole lower the surface tension of skin that enable better penetration into fungal cell wall. As a result, ergosterol synthesis is inhibited. Microemulsions are more stable and enhance better penetration and absorption than the older ways of drug delivery.

\section{MISCELLANEOUS DRUG DELIVERY SYSTEM}

Transungual drug delivery is a transport system of topical drug therapy mainly across the nail. Nail lacquers, formerly a cosmetic, has been transformed into another new approach. Drugs such as Miconazole, Ketoconazole and Ciclopirox are incorporated and marketed as medicated nail lacquer preparations. The principle works like a drug depot. The film formed on the nail plate after evaporation of solvent gives hydration and allows optimum drug release across the nail. In the onychomycosis treatment, it has the benefits of localised action, raised adherence and reduced systemic side effects. It also gives prolonged effect as single application lasts for seven days. In addition, the preparation is comparatively simple and not costly. However, it does have the issue of viscosity modification and limited drug permeability which might not reach the desired therapeutic effect. There are approaches in nail penetration that categorised into chemical, physical and mechanical methods. Chemical methods involve mercaptans, enzymes, organic solvents and keratolytic agents. Physical methods are etching, microneedles, carbon dioxide laser, electro pulsation and iontophoresis. Nail avulsion and abrasion treat nail disorder mechanically.

On the other hand, buccal mucoadhesive drug delivery system acts as a medium for the formulations to have close contact with the adsorptive mucosal surface of the oral cavity for the oral candidiasis treatment. Mucoadhesive buccal films (matrix type and reservoir type) and adhesive tablets are the dosage forms. The therapeutic agents administered includes fluconazole and nystatin. For best absorption, the partition coefficient ranges from 40 to 20000 and pKa from 2 to 10 . The thin membrane rich with blood capillary network facilitates the absorption through passive diffusion into the blood circulation. It bypasses the firstpass hepatic metabolism, preventing the premature drug inactivation. High molecular weight polymers with hydrophilic groups providing hydrogen bonding give an additional benefit in bioadhesion. It helps in adherence and to withstand salivation. ${ }^{14}$

Mucoadhesive dosage forms are easy to administer and stop the treatment. Localised target in oral cavity enables dose reduction. However, drugs with appalling odour and taste, or require large doses of administration (more than $25 \mathrm{mg}$ ) are not suitable. Salivation and swallowing problems are the limitations.

\section{DISCUSSION}

In the Vesicular Drug Delivery System (VDDS), although liposomes and niosomes shown a number of advantages in delivery of antifungal agents, some disadvantages present also restrict their use in the medical field and different formulations are given in Table 1. The most common concern regarding liposome vesicles is that high possibilities are subject to hydrolysis or oxidative degradation of the phospholipid bilayer by external triggers such as temperature or $\mathrm{pH}$ which contribute to both physical and chemical instability and consequently causes drug leakage. Besides, following systemic administration of liposomal vesicles, it will be uptake mostly by liver which exhibits largest capacity among other reticuloendothelial system (RES) organs followed by spleen for liposomal uptake. The vesicles are recognized as foreign particles in the body and undergo clearance by the macrophages and phagocytic cells. However, research studies reveal that "conjugation of PEG polymers 
with liposomal membrane may improve circulation times and prevent removal by the RES through steric stabilization". Lastly, there is also a common problem with large scale production of liposomes due to its complicated preparation steps which is recommended to prepare in laboratory level. ${ }^{15,16}$

Next, nanoparticles overcome a lot of problems in delivery of antifungal drugs in traditional methods such as reduced drug stability, poor drug pharmacokinetic, limited penetration through the tissues, poor aqueous solubility, reduced drug efficacy and side effects. As mentioned above, liposomes is the first nanoparticle introduced which is phospholipidbased at the beginning. However, due to its poor penetration through the stratum corneum, deformable liposomes are then introduced. ${ }^{17}$ For polymeric nanoparticles, their sustained released property enhanced the drug efficacy and reduced the drug toxicity. However, the polymer can be internalized by the macrophages and cause cytotoxic effects when degradation occurs. Solid lipid nanoparticles are then introduced as an alternative to polymeric particles. However, its disadvantages are low drug loading capacity and drug expulsion during storage. So, then a nanostructured lipid carrier (NLCs) is introduced. The specific lipid and oil proportions make it have better drug loading, drug released and stability. ${ }^{18}$ Amphotericin-B loaded nanofibers and liposomes are developed and even certain plant extracts are using as an antifungal drug which is safer to use the future trends used for the study are Echinocandins, the latest class of drug used for the antifungal and drug act as a fungicidal that has low drug-drug interaction which makes first-line treatment for invasive candidiasis particularly. ${ }^{19}$

Microparticulate encoded drugs in polymer coating have protected drug from degradation before reaching target tissues and hence increases the bioavailability of drugs. Microemulsions with the composition of aqueous and oil phases enhanced the drug delivered through topical and transdermal route. Microemulsions enhance the penetration of skin, perform sustained release and are more stable in extreme temperature. ${ }^{20}$ As compared to conventional drug delivery such as cream, the onset of action is much slower than the microemulsion. With the development of colloidal carriers, the fungal infection can be resolved in short time manner and reduce the complications.

Table 1: Liposomes and Niosomes as VDDS.

\begin{tabular}{ccc}
\hline Vesicular Formulation & Drug & Activity \\
\hline $\begin{array}{c}\text { Liposomes } \\
\text { Soya lecithin, cholesterol } \\
\text { tocopheryl acetate }\end{array}$ & Ketoconazole & $\begin{array}{c}\text { Sustained release upto } \\
24 \text { hr. }\end{array}$ \\
$\begin{array}{c}\text { Increase } \text { in-vitro drug } \\
\text { deposition on skin. }\end{array}$ \\
$\begin{array}{c}\text { Hydrogenated soy } \\
\text { phosphatidycholine } \\
\text { cholesterol, } \\
\text { distearoylphosphatidyl } \\
\text { glycerol }\end{array}$ & Amphotericin & $\begin{array}{c}\text { Deliver drug at the target } \\
\text { infection site. }\end{array}$ \\
$\begin{array}{c}\text { Niosomes } \\
\text { Span 60, stearic acid, } \\
\text { cholesterol }\end{array}$ & Nystatin & $\begin{array}{c}\text { Sustained-release system } \\
\text { through skin and enhanced } \\
\text { bioavailability with less } \\
\text { irritation. }\end{array}$ \\
Span 60, ethanol, \\
cholesterol
\end{tabular}

In the management of onychomycosis, a study on transungual delivery for established antifungal proved that miconazole-loading medicated nail lacquer succeeded to give extended drug release for treatment. The statement explaining medicated nail lacquer has the advantage of being not easily removed and better absorption as compared to conventional formulations can be evidenced by an investigation finding by Hafeez et al. on comparison of ketoconazole in the formulations of lacquer and cream. For efficacy and safety, an ex-vivo penetration study was conducted using nail lacquers, solution, hydrogel and colloidal carriers of antifungal drugs on nails. The results showed colloidal carriers have the best penetration rate, followed by nail lacquers. The hydrogel was least diffusible and revealed the most lag time. Systemic therapy employed together with topical therapy suggests a better treatment outcome. ${ }^{21}$

Mucoadhesive dosage forms have the property of longer residence time by holding in between gums and cheeks instead of swallowing as compared to conventional oral solution and chewable tablets. Within buccal cavity, buccal delivery is more likely for retentive, systemic transmucosal delivery whereas sublingual delivery for rapid drug release. ${ }^{22}$ Mucoadhesive drug delivery systems are suitable for drugs that have a localized effect, undergo extensive pre-systemic metabolism, narrow absorption window and narrow therapeutic index. Mucoadhesive drug delivery systems with customizable release profiles have recently gained considerable interest among formulation scientists to improve clinical outcomes of drugs. ${ }^{23}$ Mucoadhesive liposomal gel sertaconazole nitrate-loaded cationic liposomes showed a significant reduction in the microbial count with a subsequent reduction in inflammatory responses with the lowest histopathological change compared with conventional gel. $^{24}$

\section{CONCLUSION}

In conclusion, fungal infections are an issue that happens in the whole world and it can be a life-threatening disease for the immune compromised patient. Since our medical system over-reliance on antibiotics to fight the fungal infections for a long time, a lot of other drugs lost their effectiveness in treating fungal infection. Therefore, novel drug delivery systems such as vesicular drug delivery systems, nano-particulate based drug delivery systems, colloidal carriers and miscellaneous delivery systems are introduced. These systems mainly help in reducing the toxicity and increase the efficacy of antifungal drugs and thus increase the therapeutic effect in antifungal drug treatment.

\section{ACKNOWLEDGEMENT}

The authors acknowledge Faculty of Pharmaceutical Sciences, UCSI University.

\section{CONFLICT OF INTEREST}

The authors declare no conflicts of interest.

\section{ABBREVIATIONS}

VDDS: Vesicular Drug Delivery Systems; SLNs: Solid lipid nanoparticles; RES: Reticuloendothelial system; PEG: Polyethylene glycol; NLCs: Nanostructured lipid carriers.

\section{REFERENCES}

1. Mahendra P. Morbidity and Mortality Due to Fungal Infections. J Appl Microbiol Biochem. 2017; 1(1):1-2

2. Sawant B, Khan T. Recent advances in delivery of antifungal agents for therapeutic management of candidiasis. Biomed Pharmacother. 2017;96:147890.

3. More SB, Nandgude TD, Poddar SS. Vesicles as a Tool for Enhanced Topical Drug Delivery. Asian J Pharm. 2016;10(3):S196-208. 
4. Voltan AR, Quindos G, Alarcon KPM, Fusco-Almeida AM, Mendes-Giannini MJS, Chorilli M. Fungal Disease: Could Nanostructured Drug Delivery System be a Novel Paradigm for Therapy?. Int J Nanomed. 2016;11:3715-30.

5. Buchiraju R, Nama S, Sakala B, Chandu BR, Kommu A, Yedulapurapu N. Vesicular Drug Delivery System: An Overview. Res J Pharm, Biol Chem Sci. 2013:4(3):463-73.

6. Ashara KC, Paun JS, Soniwala MM, Chavda JR, Nathawani SV, Mori NM, et al. Vesicular Drug Delivery System: A Novel Approach. Mintage J Pharm Med Sci. 2014;3(3):1-14.

7. Wissing SA, Kayser O, Muller RH. Solid lipid nanoparticles for parenteral drug delivery. Adv Drug Deliv Rev. 2004;56(9):1257-72.

8. Madni A, Sarfraz M, Rehman M, Ahmad M, Akhtar N, Ahmad S, et al. Liposomal Drug Delivery: A Versatile Platform for Challenging Clinical Applications. J Pharm Pharm Sci. 2014;17(3):401-26.

9. Muller RH, Mader K, Gohla S. Solid Lipid Nanoparticles (SLN) for controlled drug delivery: A review of the state of the art. Eur J Pharm Biopharm. 2000;50(1):16177.

10. Lengyel M, Szabo NK, Antal V, Laki AJ, Antal I. Microparticles, Microspheres and Microcapsules for Advanced Drug Delivery. New Insights into Drug Delivery and Absorption. Scientia Pharmaceutica. 2019;87(3):20

11. Prajapati VD, Jani GK, Kapadia JR. Current knowledge on biodegradable microspheres in drug delivery. Expert Opin Drug Deliv. 2015;12(8):1283-99.

12. Albertini B, Passerini N, DiSabatino M, Vitali B, Brigidi P, Rodriguez L. Polymerlipid based mucoadhesive microspheres prepared by spray-congealing for the vaginal delivery of econazole nitrate. Eur J Pharm Sci. 2009;36(4-5):591-601.

13. Tang TT, Xiong BH, Liao DH, Liu XY, Xiang DX. Mechanisms of microemulsion enhancing the oral bioavailability of puerarin: Comparison between oil-in-water and water-in-oil microemulsions using the single-pass intestinal perfusion method and a chylomicron flow blocking approach. Int J Nanomed. 2013;8:4415-
26.

14. Dhobale AV Nikose $K$, Mrunal PR, Mahendra D. Recent Advances in Mucoadhesive Buccal Drug Delivery System and Its Marketed Scope and Opportunities. Int J Adv Pharm Sci. 2018;1(8):86-104.

15. Sercombe L, Veerati T, Moheimani F, Wu SY, Sood AK, Susan H. Advances and Challenges of Liposome Assisted Drug Delivery. Front Pharmacol. 2015;6:1-13.

16. Sharma VK, Mishra DN, Sharma AK, Srivastava B. Liposomes: Present Prospective and Future Challenges. Int J Curr Pharm Rev Res. 2010;1(2):1-14.

17. Gillet A, Lecomte F, Hubert P, Ducat E, Evrard B, Piel G. Skin penetration behaviour of liposomes as a function of their composition. Eur $\mathrm{J}$ Pharm Biopharm. 2011;79(1):43-53.

18. Soliman GM. Nanoparticles as safe and effective delivery systems of antifungal agents: Achievements and challenges. Int J Pharm. 2017;523(1):15-32.

19. Guruprasad BM, FamnaRoohi NK, Gowda DV. A Review on Candidiasis resistance current drug development process in its prevention and treatment. Int J Res Pharm Sci. 2020;11(1):1073-9.

20. Gungor S, Sedef EM, Aksu B. New Formulation Strategies in Topical Antifungal Therapy. J Cosmet Dermatol Sci Appl. 2012;3(1):56-65.

21. Akhtar N, Sharma H, Pathak K. Onychomycosis: Potential of Nail Lacquers in Transungual Delivery of Antifungals. Scientifica. 2016; 2016:1387936.

22. Jeremiah MC, et al. Review on Transungual Drug Delivery System. IndoAmerican J Pharm Res. 2017;7(08):686-706

23. Kumar A, Naik PK, Pradhan D, Ghosh G, Rath G. Mucoadhesive Formulations: Innovations, Merits, Drawbacks and Future Outlook. Pharm Dev Technol. 2020;1-43.

24. Abdellatif MM, Khalil IA, Elakkad YE, Eliwa HA, Samir TM, Al-Mokaddem AK. Formulation and Characterization of Sertaconazole Nitrate Mucoadhesive Liposomes for Vaginal Candidiasis. Int J Nanomed. 2020;15:4079-90.

Article History: Submission Date : 07-06-2020; Revised Date : 26-07-2020; Acceptance Date : 16-08-2020

Cite this article: Chinnappan S, Yi CL, Chen CJ, Hsia TW, Qi YH. Recent Advances in Delivery of Antifungal Agents - A Review. J Young Pharm. 2020;12(3):193-6. 